\title{
Role of long range ferromagnetic order in the electronic structure of $\mathrm{Sr}_{1-x} \mathrm{Ca}_{x} \mathrm{RuO}_{3}$
}

\author{
Ravi Shankar Singh, V.R.R. Medicherla, and Kalobaran Maiti* \\ Department of Condensed Matter Physics and Materials Science, \\ Tata Institute of Fundamental Research, \\ Homi Bhabha Road, Colaba, Mumbai 400005, India
}

(Dated: September 12, 2021)

\begin{abstract}
We investigate the role of long range ferromagnetic order in the electronic structure of $\mathrm{Sr}_{1-x} \mathrm{Ca}_{x} \mathrm{RuO}_{3}$ using high resolution photoemission spectroscopy. $\mathrm{SrRuO}_{3}$ is a ferromagnetic metal but isostructural, isoelectronic $\mathrm{CaRuO}_{3}$ is an enhanced paramagnet. Surface spectra of $\mathrm{CaRuO}_{3}$ exhibit temperature induced modifications. This is not significant in other compositions. This may be attributed to the structural changes observed in previous studies. Interestingly, the bulk spectra reveal unusual spectral changes exhibiting large decrease in the coherent feature intensity corresponding to only ferromagnetic samples, although the Ru moment is very similar in all the compositions.
\end{abstract}

PACS numbers: 75.30.Kz, 75.50.Cc, 71.45.Gm, 71.20.-b

\footnotetext{
* Author to whom correspondence should be addressed; electronic mail: kbmaiti@tifr.res.in
} 
Ruthenium based perovskite oxides have attracted a great deal of attention due to possibilities in significant technological applications in addition to various interesting fundamental issues. In particular, $\mathrm{SrRuO}_{3}$, the only itinerant ferromagnet among the $4 d$ transition metal oxides (Curie Temperature, $\mathrm{T}_{c} \sim 165 \mathrm{~K}$ ) [1], is a promising candidate for several technological applications due to its metallic character, high magnetic moment $\left(1.4 \mu_{B} / \mathrm{Ru}\right)$, high chemical stability, etc. [1, 2, 3] Ultrathin films of $\mathrm{SrRuO}_{3}$ have already been used for normal metal layers in Josephson junctions [4], spintronic devices based on spin polarized ferromagnetic tunnel junctions with ferromagnetic metal as an electrode [5], etc. Thus, ferromagnetic materials form basis for technological advances and microscopic understanding of the origin of such effect is crucial to design new materials for future applications.

Here, we investigate the role of ferromagnetic transition in the electronic structure of orthorhombically distorted perovskites (space group $\mathrm{Pbnm}$ ), $\mathrm{Sr}_{1-x} \mathrm{Ca}_{x} \mathrm{RuO}_{3}$. The average $\mathrm{Ru}-\mathrm{O}-\mathrm{Ru}$ bond angle gradually decreases from $\sim 165^{\circ}$ in $\mathrm{SrRuO}_{3}$ to about $150^{\circ}$ in $\mathrm{CaRuO}_{3}[1$, 3, 6]. Magnetic measurements exhibit ferromagnetic ground state for $x<0.8$ and enhanced paramagnetic phase for higher $x$ values [2]. Such different magnetic ground states have also been observed in ab initio calculations based on local spin density approximations (LSDA) [7, 8]. Various photoemission studies [9, 10, 11] suggest that the electron correlation strength, $U / W(U=$ Coulomb interaction strength, $W=$ bandwidth $)$ is significantly weak and similar in all the compositions. Transport measurements, on the other hand, indicate Fermi liquid behavior in $\mathrm{SrRuO}_{3}$ while $\mathrm{CaRuO}_{3}$ is non-Fermi liquid [12]. It is thus, clear that these systems exhibit varieties of interesting ground state properties, which cannot be attributed solely to the electron correlation effect. Despite numerous studies, the origin of such widely different ground state properties is still unclear.

In this letter, we report our results on the modification of the electronic structure across the magnetic phase transition in this system using state of the art high resolution photoemission spectroscopy. Experimental spectra exhibit qualitatively different bulk and surface electronic structures in all the samples and interesting evolutions with the change in temperature.

Samples were prepared by solid state reaction route followed by sintering in the pellet form for about 72 hours at $1523 \mathrm{~K}$ to achieve large grain size. Sharp features in the $x$-ray diffraction (XRD) patterns with lattice parameters similar to those in single crystalline samples [2, 6] and no signature of impurity feature indicate high quality of the samples. DC 
magnetic susceptibility, measured using high sensitivity vibrating sample magnetometer, show sharp ferromagnetic transition at $165 \mathrm{~K}$ in $\mathrm{SrRuO}_{3}$. The sharpness of the transition gradually reduces and becomes insignificant for $x \geq 0.8$. $\mu_{\text {eff }}$ for all the samples in the paramagnetic region has been estimated to be $2.8 \pm 0.2 \mu_{B}$, which is close to the theoretical spin only value of $2.83 \mu_{B}$ corresponding to $t_{2 g \uparrow}^{3} t_{2 g \downarrow}^{1}$ configuration of $\mathrm{Ru}^{4+}$ [2, 9]. Photoemission measurements were performed on in situ (base pressure $\sim 3 \times 10^{-11}$ torr) scraped sample surfaces using Gammadata Scienta analyzer, SES2002 with an energy resolution set to $4 \mathrm{meV}, 900 \mathrm{meV}$ and $300 \mathrm{meV}$ for the measurements with monochromatic He II $(40.8 \mathrm{eV})$, $\mathrm{Al} K \alpha(1486.6 \mathrm{eV})$ (twin source) and $\mathrm{Al} K \alpha$ (monochromatic source) respectively. Cleanliness of the sample surface was ensured by minimizing the higher binding energy feature in $\mathrm{O} 1 s$ spectra [13] and the absence of $\mathrm{C} 1 s$ signal. A polycrystalline silver was mounted on the same sample holder in electrical contact with other samples to determine the Fermi edge, $\epsilon_{F}$.

In Fig. 1(a), we plot $\mathrm{Ru} 4 d$ He II spectra after subtracting the $\mathrm{O} 2 p$ contributions appearing at higher binding energies. All the spectra exhibit an intense, broad feature at $1.2 \mathrm{eV}$ along with finite intensity at $\epsilon_{F}$. While the intensities at $\epsilon_{F}$ correspond well to the band structure results (termed as coherent feature), signature of the dominant contributions at $1.2 \mathrm{eV}$ is not present in these results [7, 8]. The $300 \mathrm{~K}$ spectra for all the values of $x$ are very similar exhibiting weak coherent feature intensity suggesting metallic phase in these materials. $\mathrm{Ru}$ $4 d$ He II spectra at $20 \mathrm{~K}$ exhibit significant decrease in intensity of the coherent peak, when it is normalized by the intensity at $1.2 \mathrm{eV}$ of $300 \mathrm{~K}$ spectrum. The difference in intensity between the spectra at room temperature and $20 \mathrm{~K}$ spectra gradually increases with the increase in $x$.

Since, the surface contribution is significantly large $(\sim 80 \%)$ in the He II spectra, we probe the influence of the temperature on the $\mathrm{Ru} 4 d$ contributions in the $\mathrm{Al} K \alpha$ spectra of the valence band, where the surface sensitivity is reduced to about $\sim 40 \%$. All the spectra are shown in Fig. 1(b) after normalizing by the intensity at $1.5 \mathrm{eV}$. The lineshape of the Ru $4 d$ spectra is significantly different from the He II spectra shown in Fig. 1(a). The $300 \mathrm{~K}$ spectrum of $\mathrm{SrRuO}_{3}$ exhibits intense coherent peak around $0.5 \mathrm{eV}$ and an asymmetry towards higher binding energies. Interestingly, corresponding $20 \mathrm{~K}$ spectrum exhibits significant lowering in intensity compared to the intensity at $1.5 \mathrm{eV}$. The difference in coherent feature intensity is significantly large in $\mathrm{SrRuO}_{3}$ and becomes almost insignificant in $\mathrm{CaRuO}_{3}$. This 
temperature induced modification is strikingly different from that observed in the He II spectra. This is verified in the high resolution spectra of end members shown in Fig. 1(b). The high resolution spectra of $\mathrm{SrRuO}_{3}$ exhibits a large decrease in the coherent feature intensity with the decrease in temperature, while $\mathrm{CaRuO}_{3}$ spectra remain unchanged.

In order to understand the contrasting spectral changes in the He II and $\mathrm{Al} K \alpha$ spectra, we extract the surface and bulk spectral functions from these two sets of spectra at all the temperatures for all the samples. Photoemission intensity can be expressed as $I(\epsilon)=$ $\left[1-e^{-d / \lambda}\right] F^{s}(\epsilon)+e^{-d / \lambda} \cdot F^{b}(\epsilon)$, where $d$ is the thickness of the surface layer and $\lambda$ is the escape depth of the photoelectrons. $F^{s}(\epsilon)$ and $F^{b}(\epsilon)$ represent the surface and bulk spectra, respectively. Using the values of $d / \lambda$ from Ref. [8], we have extracted the surface and the bulk contributions as shown in Fig. 2(a) and Fig. 2(b), respectively. The surface spectra exhibit dominant contributions at $1.2 \mathrm{eV}$ binding energy. The threefold degeneracy of the $\mathrm{Ru} 4 d t_{2 g}$ band is already lifted in the bulk electronic structure due to the distortion of the $\mathrm{RuO}_{6}$ octahedra [8]. The absence of periodicity along the surface normal will further reduce the crystal symmetry from $O_{h}$ symmetry towards $D_{4 h}$ symmetry at the surface. Thus, the feature at $1.2 \mathrm{eV}$ is often attributed to the $e_{g}$ band derived from the $t_{2 g}$ band due to such symmetry breaking [9, 14].

The coherent feature intensity is significantly weak in the surface spectra of all the compositions. The decrease in temperature down to $20 \mathrm{~K}$ does not lead to significant change in the surface spectra of $\mathrm{SrRuO}_{3}$ and $\mathrm{Sr}_{0.7} \mathrm{Ca}_{0.3} \mathrm{RuO}_{3}$. This is also evident in the high resolution spectra of $\mathrm{SrRuO}_{3}$. Small change in lineshape is observed for higher $x$ values, which is most significant in $\mathrm{CaRuO}_{3}$ exhibiting a large reduction in coherent feature intensity with the decrease in temperature as clearly visible in the high resolution spectra of $\mathrm{CaRuO}_{3}$. Various core level studies [13, 15] indicate significant change in the lineshape suggesting temperature induced modification in structural parameters. It is already clear that the two dimensional nature, defects, reconstructions at surface play key roles in determining the surface electronic structure. Thus, the spectral modifications observed in the surface spectra may be attributed to such temperature induced changes of the surface structure.

In Fig. 2(b), we show the bulk spectra for all the $x$ values. The room temperature spectra, normalized by integrated intensity under the curve are almost similar in all the compositions. The coherent peak appears at about $0.5 \mathrm{eV}$ with the contribution of incoherent peak (the lower Hubbard band) appearing at $2 \mathrm{eV}$. This suggests that the change in Ru-O-Ru bond 
angle across the series does not introduce significant change in the electronic structure of this system. Bulk spectra at $20 \mathrm{~K}$ are shown in the same figure by normalizing the intensity of the incoherent feature. Intensity of the coherent feature in $\mathrm{SrRuO}_{3}$ is found to decrease significantly with the decrease in temperature across the magnetic phase transition. Interestingly, such lowering in coherent features intensity is clearly visible in the bulk spectra of all the compositions exhibiting long range ferromagnetic order. The spectra corresponding to $x \geq 0.8$ remain unchanged down to the lowest temperature studied.

Band structure calculations [8] for various magnetic and non-magnetic solutions suggest that in the ferromagnetic ground state, the contribution from the down spin density of states moves above the Fermi level due to the exchange coupling between the $4 d$ electronic states. Since, the coherent feature represents the density of states observed in the band structure results, the lowering of coherent feature intensity across the magnetic phase transition may be attributed to the shift of down spin spectral intensity above $\epsilon_{F}$. This shift of the down spin density of states depends on the exchange splitting, which is also reflected in the magnetic moment. This appears to explain the change in the electronic structure in ferromagnetic compositions. However, various magnetic measurements suggest similar $\mathrm{Ru} 4 d$ moment across the entire series [2, 9]. Thus, no change in the bulk spectra of paramagnetic samples is curious and opens up an interesting question in microscopic understanding of the evolution of magnetism.

The ferromagnetism is often described within two models. (a) The Stoner description [16]: the exchange splitting gradually decreases with the increase in temperature and becomes zero at the Curie temperature leading to zero magnetic moment. (b) On the other hand, a spin mixing behavior [17] leads to a reduction in spin polarization with the increase in temperature keeping the magnetic moment unchanged. The purely Stoner behavior can be ruled out since the magnetic moment exists even in the paramagnetic phase in all the compositions. If the second case is active, the spin integrated spectra should be identical in all the compositions. Thus, significant change only in the spectra of ferromagnetic compositions in this study is curious and opens up a new dimension in understanding ferromagnetism. Since, all the compounds are essentially identical except the difference in long range order, the significant modification observed in the samples having long range order naturally suggests a relation among themselves. This suggests that in addition to the intrasite exchange interactions (responsible for local magnetic moments), intersite exchange 
correlations, which give rise to long range order presumably play a key role in determining the spectral functions observed by photoemission spectroscopy. We hope, this study will help to initiate further efforts in this direction to understand this effect in ferromagnetic materials.

In summary, we investigate the change in the electronic structure across the magnetic phase transition in a series of compounds exhibiting magnetic ground states ranging from ferromagnetic to enhanced paramagnetic. Although the intrasite exchange interactions are similar in all the compositions, the bulk spectra exhibit significant modification in the lineshape across the Curie temperature in ferromagnetic materials, while the spectra in paramagnetic samples remain unchanged down to the lowest temperature studied. This suggests that intersite exchange interactions responsible for long range order presumably play an important role in determining the electronic structure of these interesting materials. 
[1] J. J. Randall and R. Ward, J. Amer. Chem. Soc. 81, 2629 (1959); A. Callaghan, C. W. Moeller, and R. Ward, Inorg. Chem. 5, 1572 (1966); J. M. Longo, P. M. Raccah, and J. B. Goodenough, J. Appl. Phys. 39, 1327 (1968).

[2] G. Cao, S. McCall, M. Shepard, J. E. Crow, and R. P. Guertin, Phys. Rev. B 56, 321 (1997).

[3] R. S. Singh, P. L. Paulose, and K. Maiti, Solid State Physics (India) 49, 876 (2004).

[4] S. C. Gausepohl, M. Lee, L. Antognazza, and K. Char, Appl. Phys. Lett. 67, 1313 (1995).

[5] K. S. Takahashi, A. Sawa, Y. Ishii, H. Akoh, M. Kawasaki, and Y. Tokura, Phys. Rev. B 67, $094413(2003)$.

[6] Kobayashi, H., M. Nagata, R. Kanno, and Y. Kawamoto, Mater. Res. Bull. 29, 1271 (1994).

[7] D.J. Singh, J. Appl. Phys. 78, 4818 (1996); I.I. Mazin and D.J. Singh, Phys. Rev. B 56, 2556 (1997).

[8] K. Maiti, Phys. Rev. B 73, 235110 (2006).

[9] K. Maiti and R. S. Singh, Phys. Rev. B 71, 161102(R) (2005). In this paper, $\mathrm{CaRuO}_{3}$ was described to be antiferromagnetic due to (-ve) $\theta_{P}$ derived from the susceptibility in the paramagnetic region. However, recent studies suggest an enhanced paramagnetic phase in this compound.

[10] K. Maiti, R. S. Singh, and V. R. R. Medicherla, Europhys. Lett. 78, 17002 (2007).

[11] M. Takizawa, D. Toyota, H. Wadati, A. Chikamatsu, H. Kumigashira, A. Fujimori, M. Oshima, Z. Fang, M. Lippmaa, M. Kawasaki, and H. Koinuma, Phys. Rev. B 72, 060404(B) (2005)

[12] L. Klein, L. Antognazza, T. H. Geballe, M. R. Beasley and A. Kapitulnik, Phys. Rev. B 60, 1448 (1999); P. Khalifah, I. Ohkubo, H. Christen and D. Mandrus, Phys. Rev. B 70, 134426 (2004); Y. S. Lee et al., Phys. Rev. B 66, 041104(R) (2002).

[13] R. S. Singh and K. Maiti, Solid State Comm. 140, 188 (2006).

[14] K. Maiti, A. Kumar, D. D. Sarma, E. Weschke, and G. Kaindl, Phys. Rev. B 70, 195112 (2004).

[15] R. S. Singh and K. Maiti, cond-mat/0605552

[16] E.C. Stoner, Proc. R. Soc. London A 154, 656 (1936).

[17] V. Korenman et al., Phys. Rev. B 16, 4032 (1977); 16, 4048 (1977); H. Capellman, Z. Phys. B 34, 29 (1979); A.J. Pindor et al., J. Phys. F 13, 979 (1983); H. Hasegawa, J. Phys. Soc. 
Jpn. 46, 1504 (1979). 


\section{FIGURE CAPTIONS}

Fig. 1: Photoemission spectra of $\mathrm{Ru} 4 d$ valence band for different values of $x$ in $\mathrm{Sr}_{1-x} \mathrm{Ca}_{x} \mathrm{RuO}_{3}$ at $300 \mathrm{~K}$ (closed circle) and $20 \mathrm{~K}$ (open circle) using (a) He II and (b) $\mathrm{Al} K \alpha$ radiations. The top and bottom sets in (b) are the high resolution spectra of $\mathrm{SrRuO}_{3}$ and $\mathrm{CaRuO}_{3}$ using monochromatic $\mathrm{Al} K \alpha$ source.

Fig. 2: (a) Surface and (b) Bulk spectra of $\mathrm{Sr}_{1-x} \mathrm{Ca}_{x} \mathrm{RuO}_{3}$ at $300 \mathrm{~K}$ (closed circle) and $20 \mathrm{~K}$ (open circle). Top and bottom sets are the high resolution spectra of $\mathrm{SrRuO}_{3}$ and $\mathrm{CaRuO}_{3}$, respectively. 

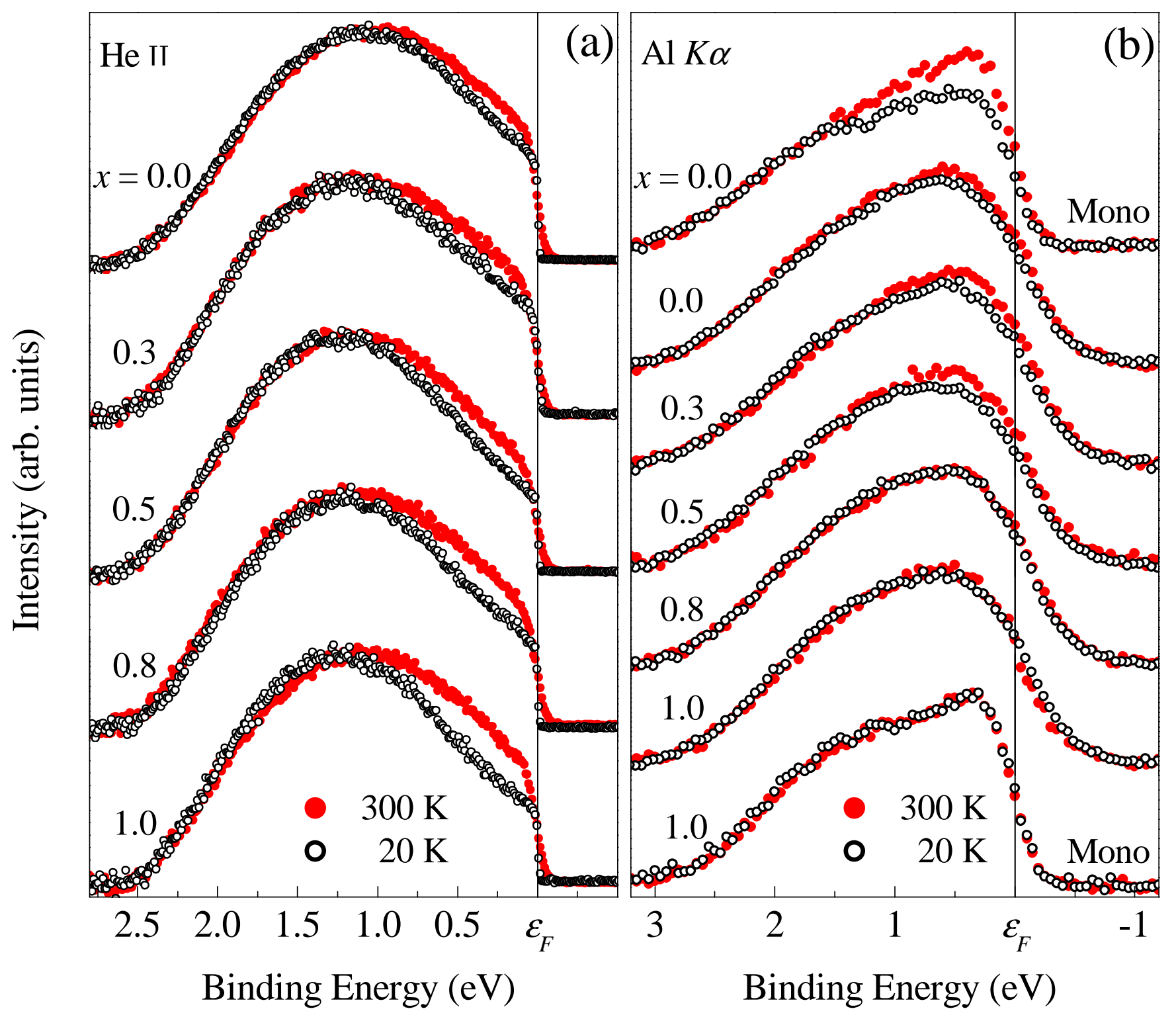


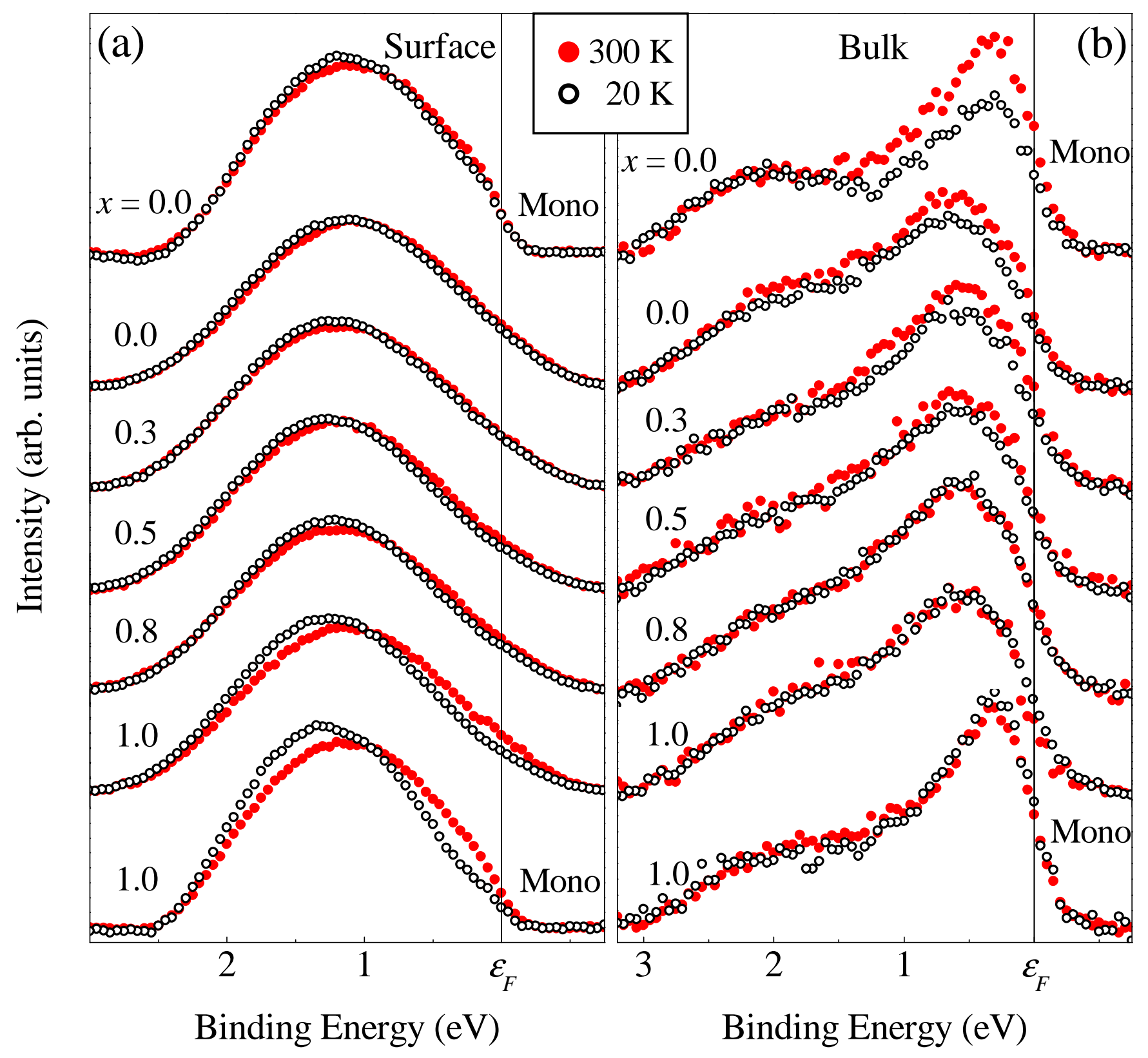

